Check for updates

Cite this: RSC Adv., 2017, 7, 22788

\title{
A strategy to speed up formation and strengthen activity of biofilms at low temperature $\dagger$
}

\begin{abstract}
Huizhi Hu, ${ }^{a}$ Junguo He, (D) *a Huarong Yu, ${ }^{a}$ Jian Liu ${ }^{b}$ and Jie Zhang ${ }^{a}$
The start-up period of biofilm reactors often takes a long time to obtain a mature and stable biofilm, especially at low temperature. We performed a long-term study to investigate a strategy to improve biofilm formation and activity at low temperature (about $14{ }^{\circ} \mathrm{C}$ ). A $25 \%$ increase in the speed of biofilm formation was observed with addition of a $50 \mathrm{nM} \mathrm{N}$-acylhomoserine lactone (AHL) mixture, yielding a sustained increase of more than $20 \%$ in biofilm activity and pollutant removal, finally resulting in shortening the start-up phase. Enhanced bacterial attachment to the suspended carrier's surface was confirmed with confocal laser scanning microscopy (CLSM). The low level of polysaccharides (PS)/ proteins (PN) at the end of biofilm formation with AHL addition also suggested that the effects of AHLS on PS production are more obvious than for PN. Analyses of the bacterial community by highthroughput sequencing showed a decrease in species diversity and a marked increase in most of the AHL-related microbial community members in an experimental reactor (ER). This work may offer a feasible approach to shorten the start-up phase of biofilm reactors and enhance biofilm performance at low temperature.
\end{abstract}

Received 22nd February 2017 Accepted 10th April 2017

DOI: 10.1039/c7ra02223a

rsc.li/rsc-advances early biofilm is often dislodged, further reducing reactor efficiency. Accelerating the stable formation of biofilm will shorten the start-up time and increase removal efficiency of a reactor in cold areas, significantly improving the wastewater treatment process.

Biofilm is a highly structured microbial community in which functionally synergistic microbes are frequently selfimmobilized. Although biofilm formation is a multiple-step process and determined by a variety of physicochemical forces (hydrodynamic force, diffusion force, gravity force, etc.) and biological forces (production of extracellular polymer, growth of cellular clusters, metabolic changes, etc.), ${ }^{4}$ aggregation of microbes can be the key to the biofilm development. Biofilm formation and behavior depends not only on environmental conditions but also on bacterial species modifying their behavior in a coordinated fashion by a type of cell-to-cell signaling known as quorum sensing (QS). ${ }^{5}$ The signals are synthesized by bacteria and detected as a means to gauge population density. When the population density reaches a threshold, signal molecules regulate gene expression. Structurally, QS signal molecules fall into a wide range of chemical classes including oligopeptides, $\mathrm{N}$-acylhomoserine lactones (AHLs), and autoinducer-2s (AI-2). QS auto-inducers in Gram negative bacteria generally belong to the AHL family. ${ }^{6}$ Previous studies have indicated that AHLs are strongly associated with the processes of biofilm formation. ${ }^{7,8}$ With appropriate molecular structures AHLs are therefore likely to improve microbial activity, and regulate extracellular polymeric substances (EPS) production, thus improving reactor performance. ${ }^{9}$
${ }^{a}$ School of Municipal and Environmental Engineering, Harbin Institute of Technology, 73 Huanghe Road, Harbin, China. E-mail: junguohe@263; Fax: +86-0451-86289099; Tel: +86-0451-86289099

${ }^{b}$ Central and Southern China Municipal Engineering Design and Research Institute Co., Ltd., 41 Jiefang Park Rord, Wuhan, China

$\dagger$ Electronic supplementary information (ESI) available. See DOI: 10.1039/c7ra02223a 
An ongoing question is if AHL effects are significant in complex culture biofilm in water, and wastewater treatment systems at the launch stage. In such systems, initial biofilm formation and subsequent biofilm behavior may be mediated by non-AHL species due to not all of the community members are capable of QS. Thus, it is important to elucidate the role of AHLs and AHL-related bacteria in biofilm formation process. Previous studies have focused primarily on AHL production in pure cultures, or the role of AHLs in microbial communities. ${ }^{\mathbf{1 0 , 1 1}}$ In contrast, little is known about exogenous AHL signals facilitating biofilm formation in complex communities, and to our knowledge, few prior publications have studied the relationship of artificially strengthening QS and biofilm formation in the initial phase at low temperature. Although the role of AHLs on biofilm formation has been characterized, the effect of low temperature on AHLs mediating the attached-growth and formation efficiency of biofilm with a complex microbial community needs a deeper understanding.

Previously, we performed a long-term study to investigate the links between AHLs and the organization and composition of complex microbial communities when biofilm develop. ${ }^{12}$ Further research found that the concentration of AHLs regulate the activity of biofilm, characteristics of sludge, and increases pollutant removal performance in mature biofilm systems. ${ }^{13}$ In the present study, a laboratory-scale sequencing batch biofilm reactor (SBBR) operating at $14{ }^{\circ} \mathrm{C}$ was dosed with AHLs at the start of reactor running, to determine the impact of exogenous AHLs on biofilm formation in the initial phase at low temperature. The aims of this research were to gauge the importance of exogenous AHLs in biofilm formation at low temperature, thus providing a strategy for achieving strengthened biofilm activity and reduced start-up times in cold areas.

\section{Materials and methods}

\subsection{Bioreactor operation}

In this study two identical sequencing batch biofilm reactors were constructed. The reactors consisted of polypropylene sphere fillers (diameter of $80 \mathrm{~mm}$, mean specific surface area of $350 \mathrm{~m}^{2} \mathrm{~m}^{-3}$ and specific weight of $8.3 \mathrm{~kg} \mathrm{~m} \mathrm{~m}^{-3}$ ) and were operated for 120 days at low temperature. Temperature was maintained approximately at $14{ }^{\circ} \mathrm{C}$ by a thermostat (GDH-4006, SCIENTZ) with circulating cool water. Next, $50 \mathrm{nM}$ AHL mixture was added to SBBR-1 (ER) at each day during the feeding phase, and no AHLs were added to SBBR-2 which was a blank control (BC). Four kinds of AHL $\left(\mathrm{C}_{6}-\mathrm{HSL}, \mathrm{C}_{8}-\mathrm{HSL}, 3-\mathrm{oxO}-\right.$ $\mathrm{C}_{12}$-HSL and $\mathrm{C}_{14}$-HSL) mixed together with the same concentration (50 nM). Each AHL was dissolved in deionized water and stored at $-20{ }^{\circ} \mathrm{C}$. Common QS signal threshold values are in the nanomolar range, although higher threshold concentrations have been reported. ${ }^{14}$ The types added were determined based on the AHL types in the BC and the concentration was based on the results of our previous research. The adding AHL are equivalent to $17.2-44.4 \mu \mathrm{g} \mathrm{L}^{-1}$ of COD. The bioreactor operation involved an $8 \mathrm{~h}$ cycle with two continuous phases of feeding $(0.25 \mathrm{~h})$, anaerobic $(1.75 \mathrm{~h})$, aerobic $(4 \mathrm{~h})$, anoxic $(1 \mathrm{~h})$, settling $(0.75 \mathrm{~h})$, and decanting $(0.25 \mathrm{~h})$. Seeding sludge used in this study was taken from the returned sludge of settling tank in Harbin wastewater treatment plant (Harbin, China). The sludge traits: sludge volume index (SVI) was 78, mixed liquor suspended solids (MLSS) was $9.3 \mathrm{~g} \mathrm{~L}^{-1}$ and mixed liquor volatile suspended solids (MLVSS) was $6.5 \mathrm{~g} \mathrm{~L}^{-1}$.

A total of $6 \mathrm{~L}$ synthetic wastewater $(\dagger)$ was fed to the bioreactor during the feeding stages and $6 \mathrm{~L}$ of treated effluent was discharged at the end of the cycle. The feed water properties were in ESI Table S3. $\uparrow$ Air was supplied through diffusers placed at the bottom of the tank. The dissolved oxygen (DO) concentrations were maintained in the range of $2-4 \mathrm{mg} \mathrm{L}^{-1}$ (aerobic period) and $0.2-0.5 \mathrm{mg} \mathrm{\textrm {L } ^ { - 1 }}$ (anoxic period), respectively, measuring with a multi-parameter water quality digital analyzer (HQ30D, HACH).

\subsection{Analytical methods}

Influent and effluent samples were collected weekly and analyzed for chemical oxygen demand (COD), ammonia nitrogen $\left(\mathrm{NH}_{4}{ }^{+}-\mathrm{N}\right)$, total nitrogen (TN), total phosphorus (TP), SVI, MLSS, and MLVSS analysis according to standard methods. ${ }^{15}$ Five milliliter aliquots of mixed sludge suspension were collected at the end of the anoxic stage and stored at $-20{ }^{\circ} \mathrm{C}$ for subsequent extracellular polymeric substances (EPS) analysis. Similarly, $1 \mathrm{~L}$ aliquots of treated effluent were kept at $-80{ }^{\circ} \mathrm{C}$ after sampling for later extraction of AHLs.

To calculate the mean biofilm weight, the samples were scraped out from the suspended carrier to a beaker to analyze weight from the second week of operation. ${ }^{\mathbf{1 2}}$

\subsection{EPS analysis}

Extraction of EPS from the sludge biomass was performed using the cation exchange resins (CER) method as described by Frolund et al. ${ }^{16}$ Polysaccharides were quantified by the phenolsulfuric acid method with glucose as the standard reference, ${ }^{17}$ and protein component was measured by the modified Lowry method ${ }^{18}$ with bovine serum albumin (Sigma-Aldrich, America) as the standard. All quantification was conducted in triplicate and averaged.

\subsection{Detection of AHLs by solid phase extraction (SPE) - ultra performance liquid chromatography/tandem mass spectrometry (UPLC-MS/MS)}

Extraction of AHLs from sludge supernatants was conducted as described by $\mathrm{Hu}$ et al. ${ }^{12}$ All samples were detected by Waters Acquity UPLC (Waters, America) coupled with mass spectrometry at a flow rate of $0.15 \mathrm{~mL} \mathrm{~min}^{-1}$. The targets were separated on BEH C18 column $(2.1 \times 50 \mathrm{~mm})$ filled with packing material with $1.7 \mu \mathrm{m}$ particle size.

The parameters of electrospray ionization were shown in ESI Table S4. $\dagger \mathrm{N}$-Hexanoyl-L-homoserine lactone ( $\mathrm{C}_{6}$-HSL), $\mathrm{N}$-octanoyl-L-homoserine lactone $\left(\mathrm{C}_{8}\right.$-HSL), $N$-(3-oxododecanoyl)-Lhomoserine lactone (3-oxo- $\left.\mathrm{C}_{12}-\mathrm{HSL}\right)$, and $N$-tetradecanoyl-DLhomoserine lactone $\left(\mathrm{C}_{14}-\mathrm{HSL}\right)$ were purchased from SigmaAldrich (America) and stored at $-20{ }^{\circ} \mathrm{C}$. 


\subsection{Genomic DNA extraction and sequencing analysis for community composition}

Total DNA was extracted from activated sludge using PowerSoil DNA Isolation Kit (MoBio, USA) according to the manufacturer's instructions. DNA quality concentrations were determined by a spectrophotometer (NanoDrop 2000, USA). Sequencing of the bacterial 16S rDNA was carried out on the Ion Torrent system by Sangon Biotech (Shanghai, China) company. The V3 hypervariable region of bacterial 16S rDNA gene was amplified using the universal primers BSF8 (5'-AGA GTT TGA TCC TGG CTC AG$\left.3^{\prime}\right)$ and BSR534 (5'-ATT ACC GCG GCT GCT GG-3') with $10 \mathrm{nt}$ barcodes (Sangon, China). PCR amplification was performed with an ABI Veriti system (Applied Biosystems, USA) according to a previous study. ${ }^{12}$

\subsection{Determining the capability and activity of the biofilm}

Dehydrogenase activity measurements were carried out according to Jurecska et $a .^{19}$ To evaluate the biofilm pollutant removal ability, $10 \mathrm{~g}$ of biofilm was washed from two reactors after the biofilm completed forming and resuspended in synthetic wastewater. The incubation temperature and operation method were the same as mentioned above in 2.1. Two bottles were incubated for four experimental cycles. The test was calculated by comparing the changes in the removal rate for $\mathrm{NH}_{4}{ }^{+}-\mathrm{N}, \mathrm{TN}$, TP and COD. Each experiment was analyzed in triplicate and data significance was evaluated with independentsamples $\mathrm{T}$ test.

\subsection{Image collection and analysis}

The three-dimensional structure of biofilm was visually monitored with confocal laser scanning microscope (CLSM, Nikon, Japan). Samples were cut into $1 \mathrm{~cm}$ length and rinsed with phosphate-buffered saline (PBS) three times, then stained with a two nucleic acid-binding stains mixture: SYTO9 $(10 \mu \mathrm{M})$ (Molecular Probes, America) and Concanavalin A (Molecular probes, America) conjugate with tetramethylrhodamine $(200 \mu \mathrm{g}$ $\mathrm{mL}^{-1}$ ). SYTO9 stains all viable bacteria in green, while Concanavalin A stains EPS polysaccharides. After 30 mins of colonization in the dark, the non-adhering stains were removed by PBS three times. Immediately after, samples were analyzed by CLSM coupled with the UltraVIEW VoX imaging system. Image analysis was performed using Volocity software (PerkinElmer, America).

\subsection{Statistical analysis}

All statistical analyses were carried out with SPSS software version 17.0 for Windows (SPSS, Chicago, IL, USA). ANOVAs was conducted to examine differences between various samples, and correlations were considered statistically significant at a confidence interval $(p<0.05)$. Every experiment was repeated 3 times for the statistical tests.

\section{Results and discussions}

\subsection{Biofilm growth process}

3.1.1 Three-dimensional structure of biofilms. Representative biofilm structures observed after four weeks using CLSM are presented in Fig. 1. We found a marked variability in threedimensional biofilm architecture between the two bioreactors. BC formed only a few, small scattered cell clusters in the week 4, while ER produced rough biofilms containing a lot of small aggregates and of variable thickness. In week 8, approximately half of suspended carrier surface were covered with flat and compact biofilms in ER, while BC developed biofilms with a patchy coverage. ER bioreactors displayed a marked and homogeneous ability to form biofilms in week 12, and produced flat and compact structures that covered the entire surface available; however, this was not observed in BC until week 16. For the bioreactor without exogenous AHLs, patchy coverage biofilms showed less EPS shrinkage, less cell adhesion and might lead to less biofilm activity, which impedes biomass immobilization. Therefore, adding AHLs remarkably improved the growth rate and microbial activity of the activated sludge, enabling sludge aggregation and provide a positive driving force for the formation of biofilm.

3.1.2 AHL was detected in two reactors. It is necessary to detect AHLs being released into the surroundings by QSregulated bacteria. Samples were collected during the formation phase, and four types of AHLs were determined from the activated sludge supernatant extract by UPLC-MS/MS. These AHLS have been widely identified in wastewater treatment bioreactors. ${ }^{20}$

$\mathrm{BC}$

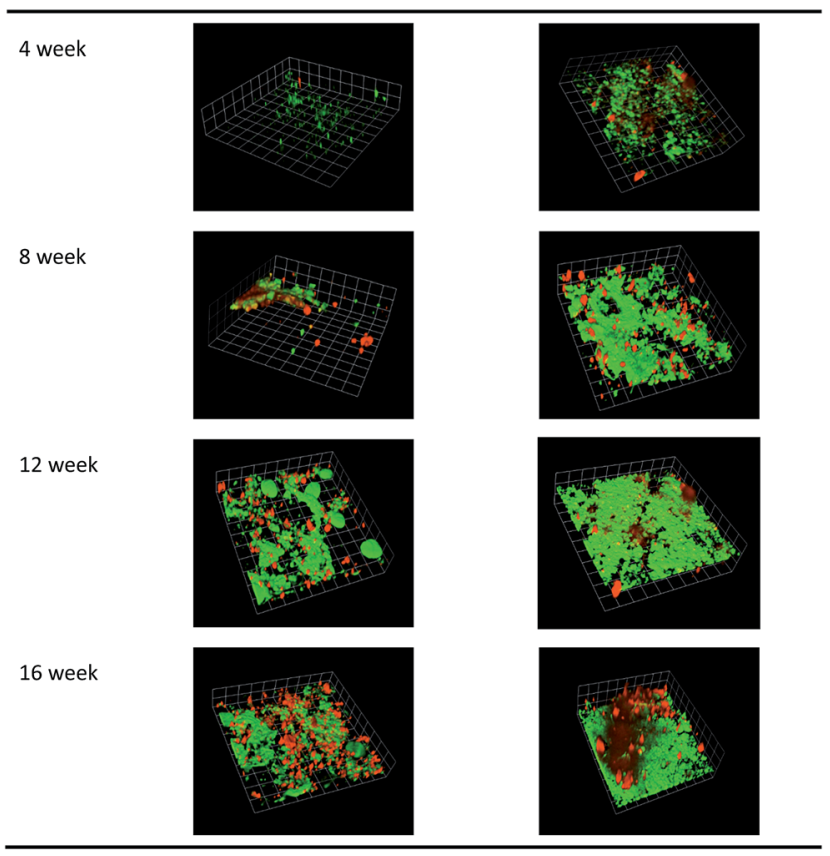

Fig. 1 Comparison of the developmental process of biofilm in two sequencing batch biofilm reactors based on microscopic visualization of structures by CLSM. Magnification time $=100 ; 1 \mathrm{grid}=73.26 \mu \mathrm{m}$; green $=$ cell; red $=$ polysaccharide. 
By comparing the spectrogram peaks of experimental samples to the control sample, we can see the peaks at the four retention times were clearly altered (Fig. 2). In BC, total concentration increased dramatically from weeks 7 and reached the maximum at 13 weeks. With the maturation of the biofilm, total concentration slightly decreased and the 4 kinds of AHLs gradually returned to a balance. In ER, however, 4 kinds of AHLs appeared in the system at the beginning of operation, which may be due to the addition of $50 \mathrm{nM}$ AHL mixture. A significant increase was produced from week 4 (27.6 nM), and reached maximum growth at week 10 (138.65 nM); a more than 4-fold increase, and 3 weeks earlier compared to BC. It is worth noting that although each AHL was added to a final concentration of $50 \mathrm{nM}$, the measured concentrations were all below $50 \mathrm{nM}$ before week 6 . This clear decrease of AHLs might be related to the degradation process or inhibition of AHL receptors in the bioreactor, named quorum quenching (QQ). ${ }^{21}$ A previous study has demonstrated that QS and QQ effects occur simultaneously in activated sludge reactors. ${ }^{22}$ The decreased concentrations of AHLs in ER might be due to motivation of QQ processes by addition of high-dose AHLs, as the system seeks to rebalance QS and QQ levels.

3.1.3 Promotion of biofilm weight and pollutant removal efficiencies by addition AHLs. One of the main parameters for the description of biofilm systems is the biofilm weight. Samples
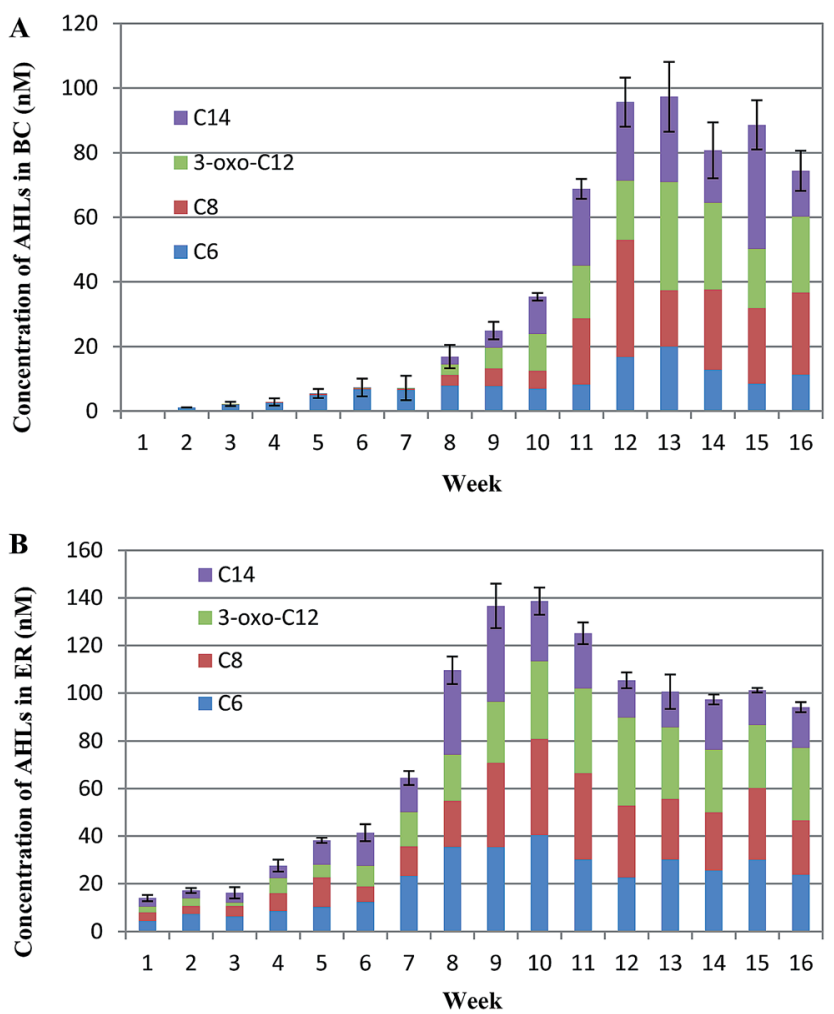

Fig. 2 The detection of $\mathrm{AHL}$ concentration in sludge supernatant sample in two SBBRs by UPLC-MS/MS. The identity and quantity of individual $\mathrm{AHLS}$ present in each sample were compared with the multiple reaction monitoring profiles of 4 standard AHLs. A total of four AHLs are shown. $\mathrm{BC}=$ blank control; $\mathrm{ER}=$ experimental reactor. Error bars are defined as standard error of the mean $(n=3$, biological replicates). were collected weekly for weight and sludge biomass analysis to monitor the formation process. Fig. 3 shows the development of the biofilm weight of two reactors. The addition of AHLs at a low concentration of $50 \mathrm{nM}$ significantly promoted biofilm formation on the suspended carrier surface. Both biofilm weights were around $70 \mu \mathrm{g} \mathrm{g}^{-1}$ carrier at the end of week 4 , which was then followed by a remarkable increase until reaching maximum values. This dramatic increase phase is characteristic of biofilm growth. Compact and stable biofilm were observed following operation of the bioreactors for 11 and 15 weeks, for ER and BC respectively. A significant difference in final biofilm weight calculated was observed between the two bioreactors $(p<0.05)$. To determine the relationship between the rapid phase (week 5 to 15 for $\mathrm{BC}$ and week 5 to 11 for ER) of biofilm weight and the time of biofilm formation, a linear-fitting procedure was performed using a linear regression analysis program. The correlation coefficient $(R)$ and determination coefficient $\left(R^{2}\right)$ of each fit line were also calculated. $R$ of ER linear fitting was higher than that of control, suggesting that adding AHLs may result in a faster rate of biofilm formation in ER compared to BC. COD values, commonly used as an indicator of organic pollutant levels in wastewater, were considerably lower for the added AHLs compared to the synthetic wastewater. The adding AHLs were equivalent to $17.2-44.4 \mu \mathrm{g} \mathrm{\textrm {L } ^ { - 1 }}$ of COD, which were only $0.004-$ $0.01 \%$ of the carbon source that apply in synthetic wastewater. However, the increased biofilm weight of ER was higher than $0.01 \%$. This indicates that the nutritional contribution of the AHLs was negligible and hence unlikely to be responsible for the rapid increase in biofilm weight. The exogenous AHL cultivation demonstrated that the attached-growth ability of the sludge could be further enhanced. These results were consistent with the biofilm structure observed by CLSM above.

The pollutant removal rates also merit consideration as AHLs dosing may have an impact on bioreactor performance. ${ }^{23}$ We quantified removal rates of $\mathrm{COD}, \mathrm{NH}_{4}{ }^{+}-\mathrm{N}, \mathrm{TN}$, and $\mathrm{TP}$ during the development of biofilm. From results reported in ESI Fig. S1, $\uparrow$ removal of COD, TN and TP were rather erratic during biofilm formation. At the beginning of biofilm formation, the mean removal efficiency of COD and nutrients were at relatively low levels $(<80 \%)$. This was followed by an increase in removal rates of COD and nutrients as biofilm formed. Stable and high

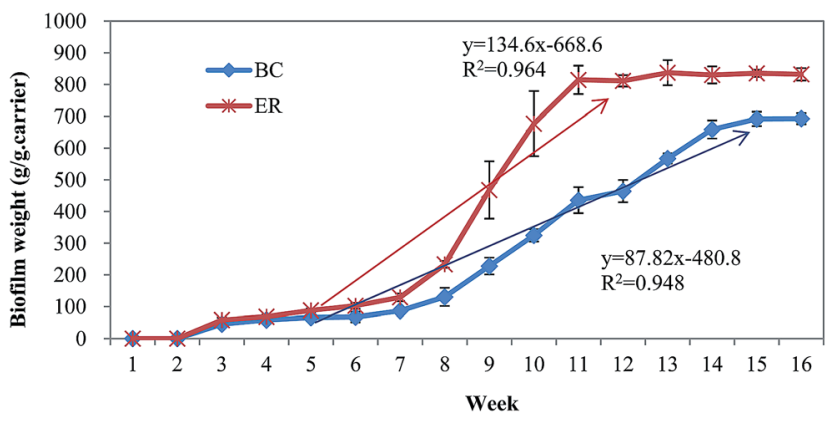

Fig. 3 Developmental process contrast of biofilm community in two sequencing biofilm batch reactors based on measurement of biofilm weight. $\mathrm{BC}=$ blank control; $\mathrm{ER}=$ experimental reactor. Error bars are defined as standard error of the mean ( $n=3$, biological replicates). 
removal efficiency for COD and nutrients were achieved once the compact biofilm was formed and had increased in biomass. Compared with the $\mathrm{BC}$, the addition of the signal molecules accelerated COD and ammonia degradation via improving the biofilm activity, indicating that the signal molecules played a clear role in enhancing removal efficiency. The removal efficiency of COD in ER increased approximately 10\% compared with $\mathrm{BC}$ at the end of experiment. Additionally, nitrification of $\mathrm{NH}_{4}{ }^{+}-\mathrm{N}$ was found to be $5 \%$ higher in ER than BC $(p<0.05)$. However, the removal performance of TP did not have significant difference between two bioreactors $(p>0.05)$, which showed that the addition of the AHLs has no positive impact on the TP removal. In addition to faster biofilm formation in ER, this indicates that quorum sensing molecules contributed to improved performance of the biofilm reactor at low temperature. Notably, in addition to a shortening in reactor start-up time by about 4 weeks, biofilms in ER were strengthened and displayed greater adhesion, resulting in improved stability and reactor efficiency. Previous study has verified that AHLs can affect bacteria communities, likely through immobilizing other functional microorganisms, and consequently, facilitate granulation and performance of reactors. ${ }^{23}$ The phenomenon in this study also indicates artificially intensified QS system may be a feasible way to regulate the bacteria behavior and promote biofilm formation at low temperature.

3.1.4 Expression of EPS correlates with biofilm formation. EPS, which are secreted by microorganisms, are binding materials for microbial floc and biofilm agglomeration. ${ }^{24}$ It is very important to clarify the roles of QS communication system in biofilm and EPS. EPS mainly consist of proteins (PN) and polysaccharides (PS). As shown in Fig. 4A and B, no significant difference was observed between the two reactors as the biomass began to transform into attached growth (from week 1 to week 3). Then, BC and ER showed a remarkable increase from week 3. Although the production of EPS largely relies on biofilm formation and microbial flocculation properties, the significant $(p<0.05)$ increase in EPS occurred before rapid growth of biofilm, with change from $73.50 \pm 5.36$ and $79.15 \pm 5.61 \mathrm{mg} \mathrm{g}^{-1}$ VSS (week 3) to $123.45 \pm 3.66$ and $153.24 \pm 6.51 \mathrm{mg} \mathrm{g}^{-1}$ VSS (week 10) for BC and ER, respectively. It means the production of EPS getting into rapid growth phase is a little earlier than biofilm weight. During the first 10 weeks, the values of PS and $\mathrm{PN}$ in ER are greater than that of in BC, and thereafter the difference narrowed. What's more, the amount of PS in ER declined from week 11, nearing the final concentration in BC. Previous studies demonstrated that AHL-mediated QS in Gramnegative bacteria is responsible for EPS production in activated sludge. ${ }^{25}$ Combined with the results above and the previous studies, it could be inferred that AHLs may affect bacteria secretion of EPS to accelerate microbial attachment and achieve the purpose of stimulating biofilm formation.

The ratio of the polysaccharide to protein ratio ( $\mathrm{PS} / \mathrm{PN}$ ) derived at different reactors was significantly different. Consistent with the EPS profile, $\mathrm{PS} / \mathrm{PN}$ increased during initial phase of biofilm formation (BC: $0.18 \pm 0.03$ (week 1) vs. $0.25 \pm$ 0.04 (week 5), $p=0.085$; ER: $0.20 \pm 0.03$ (week 1) vs. $0.32 \pm 0.01$ (week 5), $p=0.009$ ) followed by a slight fluctuation from week 6 .
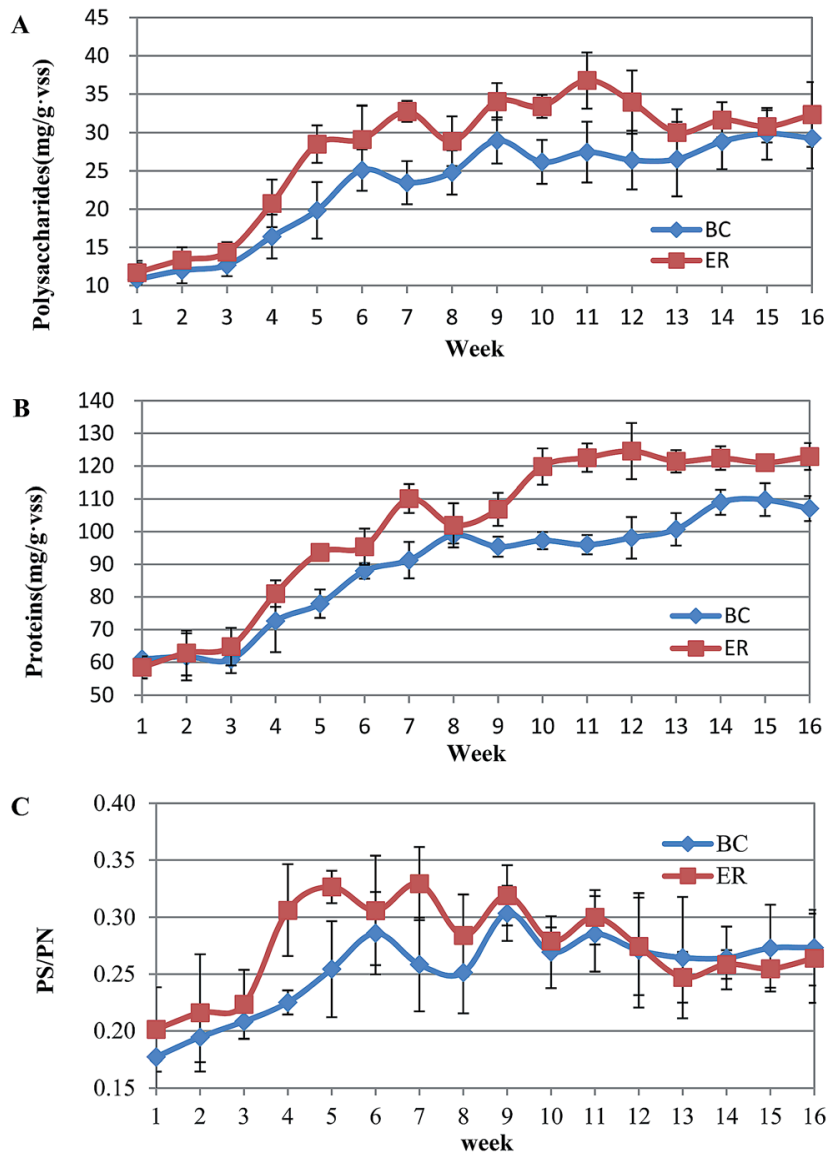

Fig. 4 Comparison of EPS in two SBBRs correlates with biofilm formation. (A): the extracellular polysaccharides (PS) and (B): extracellular proteins (PN) components. (C): the ratio of extracellular polysaccharide to protein (PS/PN). Error bars are defined as standard error of the mean ( $n=3$, biological replicates). $\mathrm{BC}=$ blank control; $\mathrm{ER}$ $=$ experimental reactor.

Specifically, a promising high level of PS/PN occurred when incubated with AHLs. ER activated sludge contained a higher PS/PN ratio and the differences were maintained until week 11. For the later phase, the $\mathrm{PS} / \mathrm{PN}$ ratio in $\mathrm{BC}$ ranged among 0.26 to 0.29 whereas that of ER decreased to around 0.25 (Fig. 4C). The significant $(p<0.05)$ difference indicated that the negative effects of AHLs on PS production are more obvious than PN. Polysaccharides are hydrophilic polymers, with the ability to absorb and exude water or biological fluids, and contribute to gel-like properties of biofilm. A high content of polysaccharide is usually viewed as being detrimental to settling and dewatering properties. Some studies have suggested that EPS composition and properties rather than the quantity, are more important in sludge flocculation. Note that in the present study although AHLs addition promoted the production of EPS, it is more likely to achieve the purpose of optimizing the biofilm structure by controlling the secretion of polysaccharide.

\subsection{Biofilm formed completely}

Although we obtained direct evidence for biofilm increase in the AHL-mediated reactor, a more crucial question about whether 
these AHLs affect biofilm characteristics and the bacteria at low temperature remained to be determined. It is very important to clarify the roles of the QS communication system in biofilm reactors. Therefore, completely formed biofilms were selected for the following batch test, and the biofilm characteristics and microbial population were compared.

3.2.1 Comparison of biofilm characteristics. The formation cycle in the two reactors was completed in week 16 , as indicated by biofilm weight and pollutant removal rates. In Table 1 , the characteristics and pollutant removal efficiencies of biofilm were almost completely different $(p<0.05)$ expect TN and TP removal rate, although the conditions in the two reactors were identical. The batch test results showed more than $30 \%$ and $15 \%$ enhancement in COD and $\mathrm{NH}_{4}{ }^{+}-\mathrm{N}$ removal rate was achieved in ER relative to $\mathrm{BC}$, respectively. However, the removal efficiency of COD and $\mathrm{NH}_{4}{ }^{+}-\mathrm{N}$ in ER increased about $10 \%$ and $5 \%$ compared to BC in section 3.1.3. This may be due to three aspects. Firstly, the biofilms were scratched from the surface of suspended carriers using a plastic plate and were cleaned gently with deionized water to remove chemical agents. In this process, some aged biofilm which is easy to fall off might be washed away. Therefore, the activity of rest of biofilm is higher. We had known the addition of AHLs significantly enhanced biofilm activity. Although we took same weight of two biofilms, the active biofilm in ER was more than that of in BC. As such, it could be inferred that the superiority of ER in removal efficiency of pollutants at batch test must be due to a relatively high activity of biofilm in ER than that of BC. Secondly, the removal efficiency of pollutants was responsible by activated sludge and biofilm in SBBR, whereas the removal efficiency of pollutants in batch test was only responsible by biofilm. So there is significant difference between two groups of data. This result also proved that introduction of AHLs in the biofilm reactors might have significant improvement on biofilm activity. Thirdly, boundary conditions and influence factors in batch test were different from SBBR. These may lead to the difference of two groups of data. The biofilm density produced with AHL addition was significantly larger than that of the control after 16 weeks ( $p$ $=0.022$ ). The mean dehydrogenase activity of the biofilm without the addition of AHL only reached $4.79 \pm 0.21 \mu \mathrm{g}$ TPF per g carrier after 16 weeks operation, compared to $5.87 \pm 0.18$ $\mu \mathrm{g}$ TPF per $\mathrm{g}$ carrier when AHLs were added. A significant improvement in the dehydrogenase activity was observed in ER. Dehydrogenase activity has been proposed as a measure of the biomass activity, which can be correlated with other parameters in wastewater treatment. ${ }^{26}$ Clearly, exogenous AHL could effectively stimulate the dehydrogenase activity, which was reasonable since the pollutant removal efficiencies in ER are much higher than BC during biofilm formation.

With the aim to further clarify the effects of exogenous AHLs on the activities of biofilm, especially whether it changes pollutants removal capacity, a batch test was explored in this investigation (Table 1). By comparing the pollutant efficiency of two reactors, we can see the values in AHL-mediated biofilm clearly increased. Biofilm performance was maintained around $27.05 \mathrm{mg}$ COD g per biofilm per day in BC, while a significant ( $p$ $=0.007$ ) increase in biofilm treatment efficiency occurred in ER, to $35.26 \mathrm{mg}$ COD g per biofilm per day. The more than $30 \%$ enhancement indicates the positive effects of AHLs on biofilm activity. The addition of AHLs also produced a notable increase $(p<0.01)$ biofilm activity on ammonia nitrogen removal from 1.82 to $2.11 \mathrm{mg} \mathrm{NH}_{4}^{+}-\mathrm{N}$ g per biofilm per day, meaning more than $15 \%$ increase. However, the TN and TP removal performance of biofilm between two reactors did not have a significant difference $(p>0.05)$, which was consistent with the study on pollutant removal efficiency above. Increasing evidence has indicated that nitrifying bacteria are strongly affiliated with quorum sensing signal molecules, especially to AHLs. Addition of AHLs to nitrifying biofilm can increase the biomass and accelerate the recovery of damaged biofilm rapidly. ${ }^{10,27}$ Furthermore, one gene ( generating a protein with high levels of similarity to $\mathrm{N}$-acyl homoserine lactone (AHL) synthase protein families in Nitrosospira multiformis. Two AHLs $\left(\mathrm{C}_{14}-\mathrm{HSL}\right.$ and 3 -oxo- $\left.\mathrm{C}_{14}-\mathrm{HSL}\right)$ were detected using an AHL biosensor and liquid chromatography-mass spectrometry (LC-MS) when nmuI, producing a LuxI homolog, was introduced into Escherichia coli. ${ }^{28}$ We also found that additional signal molecules affected the ratio of nitrifying bacteria in total bacteria in our previous experiments..$^{13}$ Consequently, it is reasonable that the addition of AHLs could improve the activity of biofilm. Then, the enhanced biofilm would contribute to improving the bioreactor performance. Additionally, adding the AHLs might just increase the activity of certain bacteria for the reason that nitrogen and

Table 1 Comparison of mature biofilm formation in blank control reactor $(B C)$ and experimental reactor $(E R)$ at $14{ }^{\circ} \mathrm{C}$

\begin{tabular}{|c|c|c|c|c|}
\hline Parameters & $\mathrm{BC}$ & ER & Increase $(\%)$ & $P$ \\
\hline Biofilm density & $448.33 \pm 17.62$ & $632.67 \pm 15.5$ & $41.21 \pm 4.61$ & 0.022 \\
\hline Total-TTC ( $\mu \mathrm{g}$ TPF per $\mathrm{g}$ carrier) & $4.79 \pm 0.21$ & $5.87 \pm 0.18$ & $22.73 \pm 3.22$ & 0.003 \\
\hline PS & $27.61 \pm 2.22$ & $30.69 \pm 1.73$ & $12.20 \pm 3.35$ & 0.01 \\
\hline $\mathrm{PN}$ & $107.08 \pm 3.28$ & $122.90 \pm 3.26$ & $14.78 \pm 0.49$ & 0.01 \\
\hline $\mathrm{PS} / \mathrm{PN}$ & $0.26 \pm 0.03$ & $0.25 \pm 0.03$ & $-2.34 \pm 4.92$ & 0.01 \\
\hline TN removal rate (mg TN g per biofilm per day) & $2.35 \pm 0.02$ & $2.57 \pm 0.03$ & $9.36 \pm 0.85$ & 0.109 \\
\hline TP removal rate (mg TP g per biofilm per day) & $0.20 \pm 0.06$ & $0.24 \pm 0.06$ & $39.14 \pm 5.64$ & 0.363 \\
\hline
\end{tabular}


COD removal rates increase apparently in AHL-medicated biofilm.

3.2.2 Microbial community structure analysis. To find a possible linkage between the performance of the reactors and quorum sensing related species composition, we monitored the microbial community shift after biofilm formation in two reactors. Species richness, coverage, and diversity estimations were calculated for each data set (Table 2). Shannon index and Chao1 index; the measures of community richness and evenness respectively, showed a community density change between two reactors. Chao1 index was slightly lower in ER than that of in BC after biofilm formation, suggesting the abundance of the microbial community is reduced after adding AHL reactor.

At the genus level, top 54 most abundant community members (relative abundance $>0.5 \%$ ) were calculated to state the changes in microbial species abundance in two reactors. The microbial community members were divided into four clusters: QQ, QS, QQ\&QS/none (bacteria can both produce and degrade quorum signal, or can neither produce nor degrade it) and others (bacteria that have not been tested in terms of its ability on quorum signal production and degradation) according to previous research. ${ }^{29,30}$ Community members in Fig. 5A showed QQ activity. $N$-Acyl homoserine lactone-degrading bacteria are very diverse and the most prevalent genus in three samples was: Brevundimonas, Microbacterium, Comamonas, Pedobacter, Mesorhizobium and Variovorax. Abundances of the QQ bacteria were significantly changed $(p<0.05)$, which suggested that the formation of biofilm has a great impact on the community structure of the reactor, with or without addition of AHLs. The Variovorax bacterium, representing cluster 1, has been isolated from soil and has been demonstrated to utilize $N$-(3-oxohexanoyl)-L-homoserine lactone as the sole source of energy and nitrogen. ${ }^{31}$ Members in Fig. 5B represented AHL-producing bacteria. Although only five genera were identified in this cluster, all the levels of them in the ER samples were considerably higher than in the blank reactor expect

Table 2 Sequencing information and diversity index under $97 \%$ similarity $^{a}$

\begin{tabular}{|c|c|c|c|c|c|}
\hline Sample ID & *Seq num & *OTU num & Shannon index & Chao1 index & Coverage \\
\hline${ }^{*} \mathrm{BC} 1$ & 11894 & 703 & 7.32 & 931.37 & 0.9821 \\
\hline BC2 & 12948 & 688 & 7.47 & 862.45 & 0.9704 \\
\hline BC3 & 12132 & 631 & 7.59 & 1001.39 & 0.9832 \\
\hline Average BC & 12324 & 674 & 7.46 & 931.74 & 0.9786 \\
\hline${ }^{*} \mathrm{ER} 1$ & 12032 & 640 & 7.69 & 838.24 & 0.9844 \\
\hline ER2 & 11384 & 760 & 8.14 & 907.11 & 0.9739 \\
\hline ER3 & 11271 & 707 & 7.67 & 849.00 & 0.9865 \\
\hline Average ER & 11562 & 702 & 7.83 & 864.78 & 0.9816 \\
\hline
\end{tabular}

${ }^{a}{ }^{*}$ Seq num $=$ sequence number; ${ }^{*} \mathrm{OTU}$ num $=$ operational taxonomic unit number; ${ }^{*} \mathrm{BC}=$ blank control; ${ }^{*}$ ER $=$ experimental reactor.

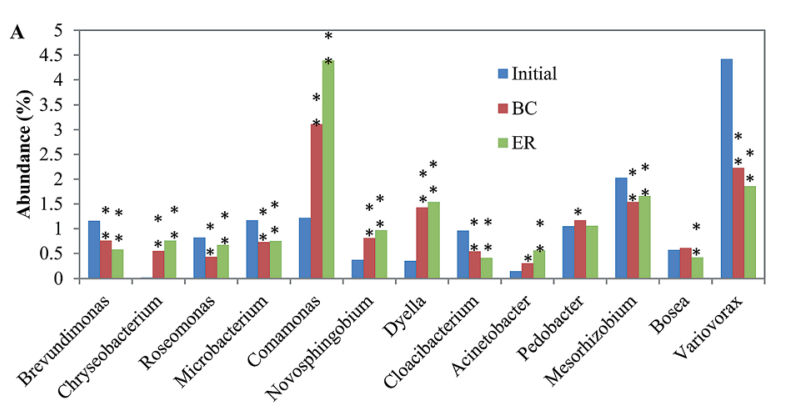

B
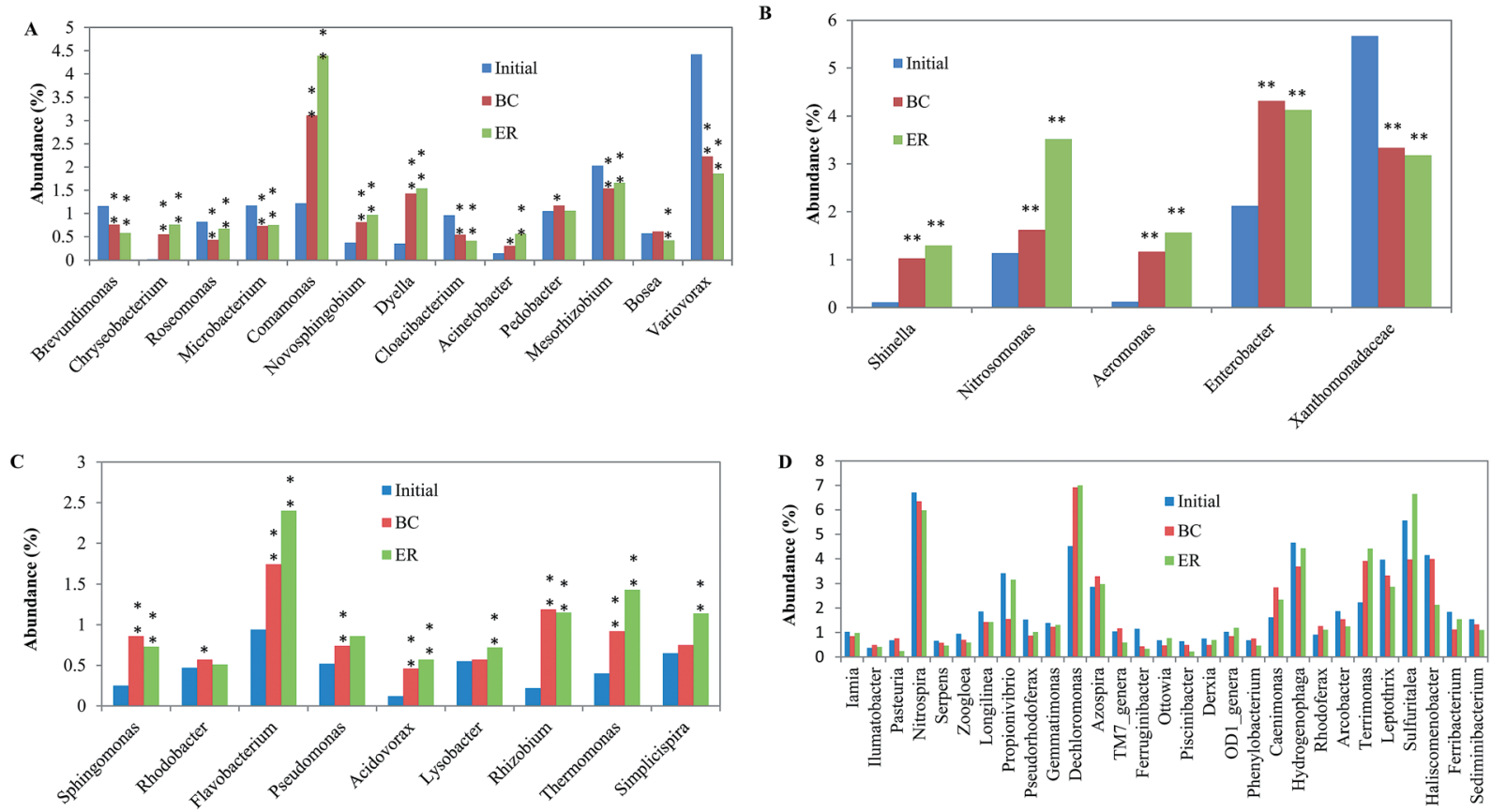

Fig. 5 Taxonomic classification of the bacterial communities at genus level. *: $p<0.05 ; * *: p<0.01$. 
Xanthomonadaceae $(p<0.01)$, which indicates that adding signal molecules positively affects AHL-producing bacteria. Microorganisms in cluster 3, which accounted for $>25 \%$ of the top 54 most abundant community members, were QQ\&QS/none bacteria (Fig. 5C). This kind of bacteria is highly diverse and falls within a large number of species among $\alpha$-, $\beta$-, $\gamma$-proteobacteria. ${ }^{32}$ AHLs addition positively affects cluster 3 bacteria for the numbers of most bacteria in ER were significantly higher than that of in the initial phase and in BC, especially for Flavobacterium, Rhizobium and Thermomonas ( $p<0.05)$. In Fig. 5D, there was no obvious trend between two bioreactors in each genus. The value of bacteria that have not been tested in terms of its ability on quorum signal production and degradation didn't increase after AHL addition. This indicated that the effect of adding AHL on QS/QQ irrelevant bacteria was not significant.

The ratios of most abundant microbial community members significantly changed after addition of AHLs. For example, Acinetobacter and Propionivibrio were found to increase ratio in the community by above $86 \%$ and $103 \%$ than that of in $\mathrm{BC}$, respectively. The addition of these AHLs also resulted in the decrease of some dominant bacteria, such as Piscinibacter and Pasteuria, for the ratio from 0.49 to 0.22 and from 0.76 to 0.24 compared with BC. The addition of AHLs was in such low concentration that the observed changes are unlikely to cause by using AHLs as a carbon and/or nitrogen source. This might be associated with AHL regulating gene expression in the reactor and the ensuing expression of the AHL-regulated phenotypes leading to changes in community composition and function. Signaling molecules were verified to have profound effects on the stability and balance of the original microbiological community. The addition of AHLs to sludge samples changed the community composition. Furthermore, exogenous signal molecular could activate bacteria by inducing the production of endogenous signal molecular by anammox cultures. ${ }^{11}$ These studies indicate that the parameters of the microbial community could be tuned through selectively manipulating AHL activity. This study also emphasizes the importance of QQ and QS as well as their roles in activated sludge system. QQ and QS are two antagonistic processes that extensively exist in the environment. When considerable quantities of AHLs were introduced, the balance of the original ecosystem was ended, and, subsequently produced a new balance. The antagonism between QQ and QS bacteria in a bioreactor provides a promising target for tuning operational performance. ${ }^{33}$ Therefore, control of QQ and QS in the system could be a promising solution for the existing problem of biofilm reactor.

\section{Conclusions}

In this study, the effect of exogenous AHLs on promoting biofilm formation at low temperature was confirmed. The addition of AHL could not only shorten biofilm formation time, but also improve the performance of biofilm. The changes in microbial community demonstrate that AHL-mediated gene expression plays a role in mediating the composition of a biofilm community by strengthening the function of quorum sensing related bacteria. The addition of exogenous AHLs also changed the balance between QS and QQ, and in this way influenced the efficiencies of the bioreactor. Our results clearly indicate that introduction of exogenous signaling molecules in biofilm reactors might provide a new approach to accelerate the biofilm start-up process and enhance biofilm performance at low temperature.

\section{Acknowledgements}

Special acknowledgement is to Huarong $\mathrm{Yu}$ for technical assistance.

\section{References}

1 S. V. Mohan, B. V. Lalit, B. Y. Vijaya and P. N. Sarma, Bioresour. Technol., 2007, 98, 1373-1379.

2 P. Falås, A. Baillon-Dhumez, H. R. Andersen, A. Ledin and J. L. Cour Jansen, Water Res., 2012, 46, 1167-1175.

3 R. Cresson, H. Carrere, J. P. Delgenes and N. Bernet, Biochem. Eng. J., 2006, 30, 55-62.

4 N. C. Boelee, H. Temmink, M. Janssen, C. J. N. Buisman and R. H. Wijffels, Water Res., 2011, 45, 5925-5933.

5 S. Raina, M. Odell and T. Keshavarz, J. Biotechnol., 2010, 148, 91-98.

6 K. Winzer, K. R. Hardie and P. Williams, Curr. Opin. Microbiol., 2002, 5, 216-222.

7 T. T. Ren, X. Y. Li and H. Q. Yu, Bioresour. Technol., 2013, 129, 655-658.

8 C. H. Tan, K. S. Koh, C. Xie, M. Tay, Y. Zhou, R. Williams, W. J. Ng, S. A. Rice and S. Kjelleberg, ISME J., 2014, 8, 1186-1197.

9 J. D. Shrout and R. Nerenberg, Environ. Sci. Technol., 2012, 46, 1995-2005.

10 X. Tang, S. Liu, Z. Zhang and G. Zhuang, Chem. Eng. J., 2015, 273, 184-191.

11 A. J. Li, B. L. Hou and M. X. Li, Bioresour. Technol., 2015, 196, 550-558.

12 H. Z. Hu, J. G. He, J. Liu, H. Y. Yu, J. Tang and J. Zhang, RSC Adv., 2016, 6, 11128-11139.

13 H. Z. Hu, J. G. He, J. Liu, H. Y. Yu and J. Zhang, Bioresour. Technol., 2016, 211, 339-347.

14 Y. L. Huang, S. Dobretsov, J. S. Ki, L. H. Yang and P. Y. Qian, Microb. Ecol., 2007, 54, 384-392.

15 APHA, Standard Methods for the Examinations for Water and Wastewater (APHA), American Public Health Association, Washington, DC, USA, 21st edn, 2005.

16 B. Frolund, R. Palmgren, K. Keiding and P. H. Nielsen, Water Res., 1996, 30, 1749-1758.

17 M. Dubois, K. A. Gilles, J. K. Hamilton, P. A. Rebers and F. Smith, Anal. Chem., 1956, 28, 350-356.

18 E. F. Hartree, Anal. Biochem., 1972, 48, 422-427.

19 L. Jurecska, K. Barkács, E. Kiss, G. Gyulai, T. Felföldi, B. Törö, R. Kovács and G. Záray, Microchem. J., 2013, 107, 108-114.

20 M. F. Siddiqui, M. Sakinah, L. Singh and A. W. Zularisam, J. Biotechnol., 2012, 161, 190-197. 
21 Y. H. Dong and L. H. Zhang, J. Microbiol., 2005, 43, 101-109. 22 X. N. Song, Y. Y. Cheng, W. W. Li, B. B. Li, G. P. Sheng, C. Y. Fang, Y. K. Wang, X. Y. Li and H. Q. Yu, Bioresour. Technol., 2014, 171, 472-476.

23 L. Li, M. Zheng, H. Ma, S. Gong, G. Ai, X. Liu, J. Li, K. Wang and X. Dong, Appl. Microbiol. Biotechnol., 2015, 99, 6471-6480.

24 L. Yang, Y. Hu, Y. Liu, J. Zhang, J. Ulstrup and S. Molin, Environ. Microbiol., 2011, 13, 1705-1717.

25 B. K. Hwang, W. N. Lee, K. M. Yeon, P. K. Park, C. H. Lee, I. S. Chang, A. Drews and M. Kraume, Environ. Sci. Technol., 2008, 42, 3963-3968.

26 A. Caravelli, L. Giannuzzi and N. Zaritzky, Water Res., 2004, 38, 2395-2405.

27 S. Batchelor, M. Cooper, S. Chhabra, L. Glover, G. Stewart, P. Williams and J. Prosser, Appl. Environ. Microbiol., 1997, 63, 2281-2286.
28 J. Gao, A. Ma, X. Zhuang and G. Zhuang, Appl. Environ. Microbiol., 2014, 80, 951-958.

29 H. Lade, D. Paul and J. H. Kweon, Int. J. Mol. Sci., 2014, 15, 2255-2273.

30 C. H. Tan, K. S. Koh, C. Xie, J. Zhang, X. H. Tan, G. P. Lee, Y. Zhou, W. J. Ng, S. A. Rice and S. Kjelleberg, Npj Biofilms and Microbiomes., 2015, 1, 15006.

31 J. R. Leadbetter and E. P. Greenberg, J. Bacteriol., 2000, 182, 6921-6926.

32 C. D. Angelo-Picard, D. Faure, I. Penot and Y. Dessaux, Environ. Microbiol., 2005, 7, 1796-1808.

33 H. R. Yu, G. R. Xu, F. S. Qu, G. B. Li and H. Liang, Appl. Microbiol. Biotechnol., 2016, 100, 7887-7897. 